\title{
Reliability and Accuracy of Astronomical Observations by Amateurs
}

\author{
Zdenek Kviz \\ School of Physics, University of New South Wales, Sydney, NSW, Australia
}

Who is an amateur-astronomer? We would certainly find several definitions if we tried to get answers from the audience. I am not trying to force anyone to accept my definition, in fact I do not know if I really have one. Someone who likes to read books about astronomy? Professional scientist, expert in other areas of science, but interested in astronomy? Science fiction writer who writes about space travel? Retired professional astronomer who is no longer paid for his work in astronomy? Constructor of telescopes or astronomical instruments? Wel1, each of these could be discussed. But we will rely, on this occasion, on common sense and take any one who is interested in astronomy and contributes to its progress either by observation or by construction of astronomical equipment and has not an official education in astronomy. He/she simply likes the idea that he/she is contributing by his/her work to our knowledge of the universe and feels proud of it.

Obviuusly every amateur likes the recognition of his/her work by professional astronomers but sometimes he/she may not be aware that his/her work may be of value not only by increasing the quality of observation but also by supplying additional information about the conditions, reliability and accuracy of the observation. Any attempt to describe meticulously all conditions of observation or to check carefully the reliability and repeatability of the results may greatly improve the quality of any amateur's work. Discovery of a comet or of a nova or supernova is an important contribution itself, but can be improved with information about the estimate of brightness and limits of accuracy of this estimate. The new supernova in the LMC - a very suitable act of celebration of the anniversary of SAF - gives us a good example. New Zealand amateur Albert Jones confirming its non-visibility the night before discovery, gave an excellent limitation on the time of the supernova's outburst.

I would like to show here two examples of how accurate observations by amateurs, or at least observations made by an amateur's means can be.

Many years ago I used to organise with my colleagues visual and telescopic observations of meteors. Fig. 1. shows the luminosity function derived for both sporadic and Perseid meteors independently by three groups of eight observers. The results are plotted separately for each group and thus the scatter of the observed values gives 


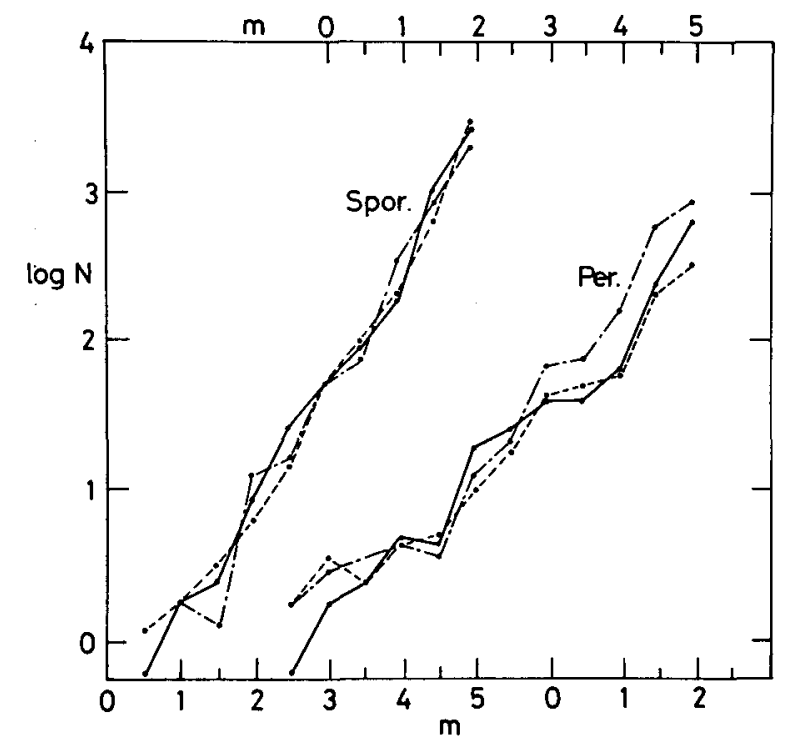

Fig.1. Luminosity function of meteors for 3 independent groups of 8 observers, separately for sporaric meteors and Perseids. Lower magnitude scale for sporadic meteors, upper scale for Perseids.

the measure of accuracy of such observations. Clearly the estimates of the brightness to one half of the magnitude are quite justified. The luminosity function was obtained using the probability of the observation of a meteor of respective brightness, the calculation of which was based on simultaneous but independent perception of a meteor by several observers. Details can be found in the original paper (Kviz 1965).

We organized various tests of observers under the supervision of a psychologist about their observational ability and reliability. Special tests concerning the alertness of the observers were also performed during the observation, to be sure that our results would be good. However, we made a mistake, we did not describe and we did not publish the precautions and tests we used. When discussing the results with a colleague I was surprised by his question "How many observers fell asleep?" He was right, how should he know anything about the reliability of our results when we did not describe the measures we did adopt? Thus I urge all amateurs to describe all details which can help someone else, who is reading the paper, to get the right idea about the reliability and accuracy of their observational results.

Now we turn to the observation of variable stars. As an example I would like to present the observation of one professional, Father O'Connell. (1951), which was made by what nowadays would be called amateur methods, visual estimates of the brightness of the variable star SV Cen based on the comparison of its photographic images with the images of neighbouring stars. Fig.2. shows the comparison of the light curve based on o'Connell's normal points and the 1 ight curve from individual photoelectric observat- 


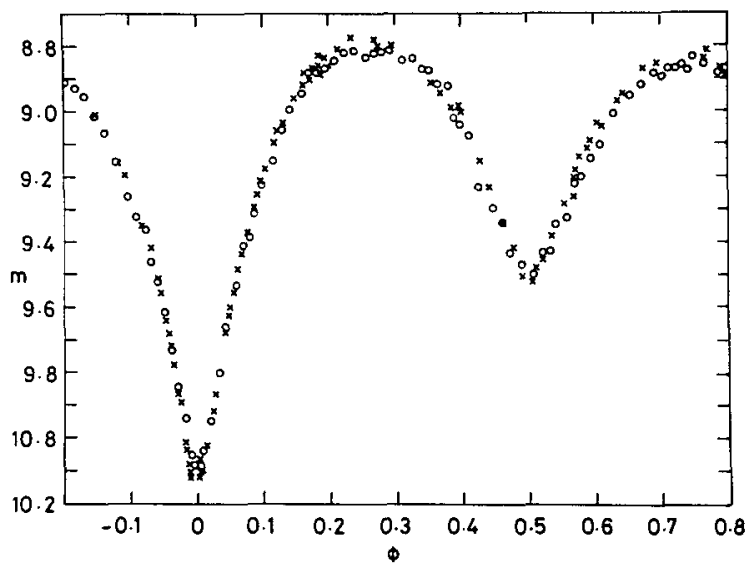

Fig. 2. Light curve of SV Centauri. o - normal points of o'Connell estimates, $x$ - individual measurements by Irwin.

ions of the same star made by J. Irwin (1972). For those, who are not familiar with the expression "normal points", I would simply explain that each normal point represents an average of many observations (estimates). The accuracy of o'Connell's observations is widely known and perhaps very few people are capable of achieving it, how ever this example shows clearly what any amateur can do if he/she is experienced, patient and diligent with a small photographic camera. These days many amateurs can afford even photoelectric photometers and proportionally better results can now be achieved with less effort. However, we should keep in mind that photographic records have still a great value as they contain information about many stars and will be even more valuable for future generations of astronomers tracing the past changes of various objects in the sky.

With observations of professional astronomers it is tacitly assumed, that all precautions concerning the proper functioning of the equipment, including the clock, and all necessary corrections were taken into account. It is considered as a professional responsibility of the author to guarantee the reliability of the results. The amatuer, espacially if he/she is not yet well known for his/her work, should put all effort into describing in detail how the observations or measurements have been done. (Sometimes even professional astronomers omit important information concerning the methods used). Take, for example, timing of the minima of eclipsing variable stars. often there are no regular observations, there are gaps in measurements, etc. Now, putting all times of minima together we may see that one minimum, perhaps quite isolated, does not fit among the others. What is the reason? Real jumps in the period changes of the star? Malfunctioning of the clock? Omission to take into account the heliocentric correction? What should an astronomer studying period changes of such a star - perhaps many years later, when the observer is no longer alive to be consulted think about that? please, preserve the proper description of your observations for future use. 
The amateurs, as the word itself, based on its Latin origin "amare - to love" indicates, love astronomy, they are not paid for their work. Some of them, as the discoverers of comets, are honoured in the way that the comets are named after them. I wish to propose, that discoverers of novae, supernovae and other variable stars should also be honoured in similar way, the stars should bear their names. There are many stars and many problems about them need to be solved. Professional astronomers having now very powerful telescopes in all spectral ranges of electromagnetic radiation are too busy with a great variety of new exciting objects. Many survey programs of professional observatories have been discontinued. By encouraging the amateurs in this way, we could gain much more information about various astronomical objects and we would be praised for that by future generations.

References:

Kviz 1965, BAC 16, 263.

O'Connell 1951, Riverview Coll. Obs. Pub. 11, 69.

Irwin J.B. \& Landolt A.U. 1972. PASP 84, 686, 